\title{
ARCHIVING ASTEROID PHOTOMETRIC DATA
}

\author{
Edward F. Tedesco \\ Jet Propulsion Laboratory \\ California Institute of Technology \\ IPAC 100-22 \\ Pasadena, CA 91125, U.S.A.
}

Current programs which produce asteroid photometric data range from astrometry through visual and near-infrared photometry and spectrophotometry to thermal infrared ( 8 to 30 micrometers) photometry. These data are published in a variety of places including observatory publications, IAU Circulars, the Minor Planet Circulars, and various journals.

In the case of thermal infrared data sometimes only the parameters (albedos and diameters) derived from the observed fluxes are published. Photometric data at standard photometric wavelength bands are nearly always published as magnitudes and/or color indices. This is sufficient for data obtained with photomultiplier tubes (PMT) as the reduction methods are standardized and have been in use for over 40 years. Where non-standard bands are used, and/or the data are obtained using Charge Coupled Device (CCD) cameras, the situation is quite different. For example, spectrophotometric data are often only published as the ratio between the asteroid flux and that of a "solar" star. In the case of such data obtained with PMTs it would be useful if the reduced magnitudes or, better yet, actual photon count rates and time, were preserved. For data obtained with CCDs it can be argued that the actual images should be preserved.

The format in which these data are preserved is also an important aspect of this issue. Frequently, past observations (especially asteroid lightcurves) have been published only in the form of figures. Until 1979 virtually all asteroid photometric data were published either as points in figures or numbers in tables. In 1979 a data base, called TRIAD (cf., B. Zellner, 1979, In "Asteroids", T. Gehrels, ed., page 1011) was established to collect and make available asteroid photometric data in machine readable form. Subsequently, other data bases have been established which contain averaged results (e.g., broad-band color indices) for each asteroid with such observations, (E. Tedesco, 1989, In "Asteroids II", R.P. Binzel, T. Gehrels, and M.S. Mathews, eds., page 997). During 1992, papers will be published containing all magnitude and UBV color indices of asteroids obtained (and published!) through 1990. Individual observations will be made available in machine-readable form thus saving future investigators the several hundred hours of effort required to extract these data from the literature.

In the future an efficient means of preserving these data in an easily retrieved form must be found. The five points outlined by Dr. Jaschek in his working paper serve as an excellent starting point. 


\section{ARCHIVING PAST ASTEROID PHOTOMETRIC DATA}

\subsection{Asteroid Lightcurve Data}

A group of two Swedish and four Italian astronomers maintain a machine-readable data base of asteroid lightcurve photometry (cf., Lagerkvist, et al., 1988, "Asteroid Photometric Catalogue; First Update", Consiglio Nazionale della Ricerche, Roma). They digitized hundreds of lightcurves for which no numerical data had been published. The current version includes 3,467 photoelectric asteroid lightcurves for 579 different asteroids published between 1949 and 1987. Included in this 1,164 page catalog are summary information and figures (all to the same scale) for each lightcurve.

\subsection{Asteroid Magnitude Observations}

Next year E. Tedesco will publish a data base containing approximately 34,000 photographic, and 5,100 photoelectric magnitudes of asteroids obtained between 1934 and 1990. The photographic magnitude portion of this data base is maintained by $B$. Marsden at the Minor Planet Center at the Smithsonian Astrophysical Observatory and the photoelectric magnitude portion by E. Tedesco at the Jet Propulsion Laboratory.

\subsection{Asteroid Broad-Band Color Observations}

In 1992 E. Bowell and E. Tedesco will publish a data base containing about 4,000 broadband asteroid color indices published between 1949 and 1990.

\section{ARCHIVING FUTURE ASTEROID PHOTOMETRIC DATA}

An asteroid lightcurve data base is maintained by the Swedish-Italian group who obtain much of these data. Some data are obtained in machine-readable form and some are still digitized from figures.

The photographic magnitudes (and some magnitudes obtained with CCD cameras) are submitted to the Minor Planet Center (MPC) as part of astrometric observations. The photoelectric observations, both magnitudes and color-indices, are culled from the literature.

Thus, for the case of asteroid photometric data obtained with photomultiplier tubes, the situation is under informal control. The case for data obtained with area detectors and at longer wavelengths is less secure.

For example, as CCDs become the photometric detectors of choice (or necessity) it would be advantageous to archive each image. The problem here is one of observatory policy and data management. It is not generally a policy of observatories in the U.S. to archive such data. Many observers are indifferent, or opposed, to this practice. There is no place to deposit such data. The media for storage, and data formats, of such data differ among observers and institutions.

Clearly this problem will not be solved overnight. In the future an efficient means of preserving these data in an easily retrieved form must be found. The five points outlined by Dr. Jaschek in his working paper serve as an excellent starting point.

Perhaps an IAU resolution encouraging such archiving would serve to stimulate action along these lines at some of the major observatories. 